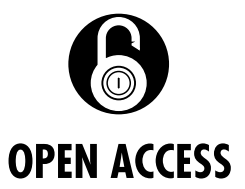

- Additional material is published online only. To view, please visit the journal online (http://dx.doi.org/10.1136/ gutjnl-2018-317263).

For numbered affiliations see end of article.

Correspondence to Dr Guy E Boeckxstaens, Department of Chronic Diseases, Metabolism and Ageing; Translational Research Center for Gastrolntestinal Disorders, Intestinal Neuroimmune Interactions, University of Leuven, Leuven 3000, Belgium; guy.boeckxstaens@kuleuven.be

NS, EL and PJG-P contributed equally.

GM and GEB contributed equally.

Received 27 July 2018 Revised 16 October 2018 Accepted 18 October 2018 Published Online First 24 November 2018

Check for updates

(c) Author(s) (or their employer(s)) 2019. Re-use permitted under CC BY-NC. No commercial re-use. See rights and permissions. Published by BMJ.

To cite: Stakenborg N,

Labeeuw E, Gomez-Pinilla PJ et al. Gut

$2019: 68: 1406-1416$

\title{
Preoperative administration of the 5-HT4 receptor agonist prucalopride reduces intestinal inflammation and shortens postoperative ileus via cholinergic enteric neurons
}

\author{
Nathalie Stakenborg, ${ }^{\oplus 1}$ Evelien Labeeuw, ${ }^{1}$ Pedro J Gomez-Pinilla, ${ }^{1}$ \\ Sebastiaan De Schepper, ${ }^{1}$ Raymond Aerts, ${ }^{2}$ Gera Goverse, ${ }^{3}$ Giovanna Farro, ${ }^{1}$ \\ Iris Appeltans, ${ }^{1}$ Elisa Meroni, ${ }^{1}$ Michelle Stakenborg, ${ }^{3}$ Maria Francesca Viola, ${ }^{1}$ \\ Erika Gonzalez-Dominguez, ${ }_{1}^{1}$ Goele Bosmans, ${ }_{1}^{1}$ Yeranddy A Alpizar, ${ }^{4}$ Albert Wolthuis, ${ }^{2}$ \\ Andre D'Hoore, ${ }^{2}$ Kim Van Beek, ${ }^{1}$ Simon Verheijden, ${ }^{1}$ Marleen Verhaegen, ${ }^{5}$ Rita Derua, ${ }^{6}$ \\ Etienne Waelkens, ${ }^{6}$ Milena Moretti, ${ }^{7}$ Cecilia Gotti, ${ }^{7}$ Patrick Augustijns, ${ }^{8}$ Karel Talavera, ${ }^{4}$ \\ Pieter Vanden Berghe, ${ }^{9}$ Gianluca Matteoli, ${ }^{3}$ Guy E Boeckxstaens ${ }^{1}$
}

\section{ABSTRACT}

Objectives Vagus nerve stimulation (VNS), most likely via enteric neurons, prevents postoperative ileus (POI) by reducing activation of alpha7 nicotinic receptor ( $\alpha 7 n A C h R)$ positive muscularis macrophages $(\mathrm{mM} \phi)$ and dampening surgery-induced intestinal inflammation. Here, we evaluated if 5-HT4 receptor (5-HT4R) agonist prucalopride can mimic this effect in mice and human

Design Using $\mathrm{Ca}^{2+}$ imaging, the effect of electrical field stimulation (EFS) and prucalopride was evaluated in situ on $\mathrm{mM} \phi$ activation evoked by ATP in jejunal muscularis tissue. Next, preoperative and postoperative administration of prucalopride $(1-5 \mathrm{mg} / \mathrm{kg})$ was compared with that of preoperative VNS in a model of $\mathrm{POI}$ in wild-type and $\alpha 7 n A C h R$ knockout mice. Finally, in a pilot study, patients undergoing a Whipple procedure were preoperatively treated with prucalopride $(n=10)$, abdominal VNS $(n=10)$ or sham/placebo $(n=10)$ to evaluate the effect on intestinal inflammation and clinical recovery of POI.

Results EFS reduced the ATP-induced $\mathrm{Ca}^{2+}$ response of $\mathrm{mM} \phi$, an effect that was dampened by neurotoxins tetrodotoxin and $\omega$-conotoxin and mimicked by prucalopride. In vivo, prucalopride administered before, but not after abdominal surgery reduced intestinal inflammation and prevented POI in wild-type, but not in $\alpha 7 n A C h R$ knockout mice. In humans, preoperative administration of prucalopride, but not of VNS, decreased $1 / 6$ and $/ / 8$ expression in the muscularis externa and improved clinical recovery.

Conclusion Enteric neurons dampen $\mathrm{mM} \phi$ activation, an effect mimicked by prucalopride. Preoperative, but not postoperative treatment with prucalopride prevents intestinal inflammation and shortens POI in both mice and human, indicating that preoperative administration of 5-HT4R agonists should be further evaluated as a treatment of $\mathrm{POI}$.

Trial registration number NCT02425774.

\section{Significance of this study}

What is already known on this subject?

- Vagus nerve stimulation (VNS), most likely via enteric neurons, prevents activation of muscularis macrophages $(\mathrm{mM} \phi)$ resulting in fastened recovery of Gl function in a murine model of postoperative ileus (POI).

- Alpha7 nicotinic receptor ( $\alpha 7 n A C h R)$ expression on the resident $\mathrm{mM} \phi$ is essential for the induction of this cholinergic antiinflammatory pathway in the gut.

What are the new findings?

- Supernatant of myenteric ganglia cultures imprints a tolerogenic phenotype and induces the expression of nicotinic acetylcholine receptors in bone marrow-derived macrophages.

- Electrical and pharmacological activation of enteric neurons modulates ATP-induced $\mathrm{Ca}^{2+}$ response in intestinal $\mathrm{mM} \phi$.

- Similar to VNS, the 5-HT4 receptor (5-HT4R) agonist prucalopride, a compound known to enhance neurotransmitter release from enteric neurons, prevents $\mathrm{mM} \phi$ activation and reduces POI, but only if administered prior to surgery.

- This anti-inflammatory effect is mediated via the $\alpha 7 n A C h R$.

- In a clinical pilot study, we showed that also in human prucalopride administered before abdominal surgery has anti-inflammatory properties and improves postoperative recovery in human.

\section{INTRODUCTION}

Each patient undergoing any abdominal surgical procedure will develop a transient episode of impaired GI motility or postoperative ileus (POI). It clinically presents as the inability to tolerate food with abdominal distension, lack of flatus 
Significance of this study

How might it impact on clinical practice in the foreseeable future?

- Our study shows that enteric neurons exert an inhibitory effect on $\mathrm{mM} \phi$ activation and indicates that preoperative activation of this pathway by prucalopride, or perhaps other 5-HT4R agonists, should be further explored as treatment of $\mathrm{POI}$ in clinical care.

and defecation. ${ }^{1}$ Although some argue that uncomplicated POI should be considered as a 'physiological' response of the intestine to a traumatic event, it clearly has a significant impact on patient morbidity with prolonged hospitalisation and thus increased costs. ${ }^{2}$ Minimally invasive surgery and the introduction of fast-track postoperative care have significantly reduced hospitalisation stay. ${ }^{1}$ Nevertheless, clinical management of POI is still restricted to conventional supportive measures consisting of nothing by mouth, intravenous fluids and prokinetics. The efficacy of prokinetics, including ghrelin agonists, metoclopramide and cisapride, to restore GI motility after surgery has however been rather disappointing. ${ }^{34}$

Most likely, the lack of effect of prokinetics can be explained by the insight that intestinal handling during surgery triggers the activation of resident muscularis macrophages $(\mathrm{mM} \phi)$ leading to inflammation of the intestinal muscle layer with influx of mainly monocytes and neutrophils. ${ }^{5-7}$ This inflammatory process is associated with impaired contractility of intestinal smooth muscle strips to cholinergic agonists in vitro and reduced intestinal transit in vivo. ${ }^{5-7}$ Administration of prokinetics in the postoperative period is therefore likely to be ineffective. Of note, however, interventions that prevent surgery-induced intestinal inflammation have been abundantly shown in preclinical models to restore GI motility. In line, POI is significantly shortened in mice lacking resident $\mathrm{mM} \phi$, such as op/op and clodronate-treated mice, indicating that $\mathrm{mM} \phi$ represent an important target to shorten POI. ${ }^{89}$ In previous studies, we confirmed this concept and showed that electrical stimulation of the vagus nerve (VNS) prior to surgery reduced surgery-induced inflammation and prevented POI in a preclinical model. ${ }^{10-12}$ This anti-inflammatory effect was mediated by acetylcholine (ACh) acting on $\alpha 7$ nicotinic receptors $(\alpha 7 \mathrm{nAChR})$ located on $\mathrm{mM} \phi .{ }^{12}$ Vagus nerve endings however do not directly interact with $\mathrm{mM} \phi$, but rather synapse with enteric neurons in close proximity to $\mathrm{mM} \phi .{ }^{13}$ Hence, we proposed that the therapeutic effect of VNS in POI most likely results from cholinergic enteric neurons dampening $\mathrm{mM} \phi$ activation.

In the present study, we aimed (1) to provide further evidence supporting this hypothesis and (2) to explore the therapeutic properties of pharmacological potentiation of this enteric neuron-mediated anti-inflammatory pathway both in mice and human. To pharmacologically potentiate neurotransmitter release from enteric neurons, we used the selective 5-HT4R agonist prucalopride (PRUC), known to enhance the release of both cholinergic and excitatory non-adrenergic non-cholinergic neurotransmitters from enteric neurons, ${ }^{14}{ }^{15}$ and used as a treatment of constipation. This compound has recently been evaluated as treatment of POI in two clinical trials with no or a small effect. In both studies, however, PRUC was administered after surgery when the contractile properties of the inflamed intestine are known to be compromised. Based on our previous work, we anticipated that to be effective, PRUC should be administered prior to surgery to stimulate the enteric neuron-mediated anti-inflammatory pathway. In the present study, we therefore first evaluated to what extent electrical and pharmacological stimulation of enteric neurons is indeed able to dampen $\mathrm{mM} \phi$ activation in vitro. Next, we compared the efficacy of preoperative and postoperative administration of PRUC with that of VNS in a model of POI and evaluated the anti-inflammatory properties of preoperative administration of PRUC and abdominal VNS in patients undergoing abdominal surgery.

\section{METHODS}

Please see online supplementary materials for an expanded version of this section.

\section{ANIMAL EXPERIMENTS}

Experiments were performed using female wild-type (C57BL/ 6JOlaHsd), $\alpha 7$ nAChR knockout (KO), CX3CR1 $1^{\text {GFP/WT }}$ and Wnt. $1^{\mathrm{GCaMP} 3}$ mice, which were bred and housed in the specific pathogen-free environment of the animal facility (KU Leuven).

\section{Cell culture experiments}

Isolation of enteric ganglia

Based on a protocol published by Grundmann et $a l^{16}$, myenteric ganglia were isolated from the small intestine of naive female wild-type mice aged 8-10 weeks. Briefly, the jejunal muscularis externa was peeled from the mucosa and digested with Liberase $\mathrm{TH}$ and DNase I for $30 \mathrm{~min}$ at $37^{\circ} \mathrm{C}$ after which individual myenteric ganglia were identified and collected using a stereotactic microscope to perform gene expression experiments.

\section{Myenteric ganglia conditioned medium collection}

Jejunal muscularis externa was peeled and enzymatically digested at $37^{\circ} \mathrm{C}$ in Alpha MEM medium (Lonza) containing 5\% fetal bovine serum (FBS) (Biowest), $100 \mu \mathrm{g} / \mathrm{mL}$ penicillin (Lonza), $100 \mu \mathrm{g} / \mathrm{mL}$ streptomycin (Lonza), $5.5 \mu \mathrm{M}$ beta-mercaptoethanol (Gibco), $250 \mu \mathrm{g} / \mathrm{mL}$ collagenase IV (Sigma-Aldrich), $500 \mu \mathrm{g} / \mathrm{mL}$ protease I (Sigma-Aldrich) and $5 \mathrm{U} / \mathrm{mL}$ DNaseI (Roche) shaking at $120 \mathrm{RPM}$ for 1 hour and trypsinised with $0.25 \%$ Trypin/EDTA (Invitrogen) at $37^{\circ} \mathrm{C}$ for $15 \mathrm{~min}$. Single cell suspension from the jejunum was seeded on coverslips coated with $0.1 \%$ poly-D-lysine (Sigma-Aldrich) and $20 \mu \mathrm{l} / \mathrm{mL}$ laminin (Sigma-Aldrich) and cultured in neurobasal-A medium (Gibco) supplemented with $10 \% \mathrm{FBS}, 100 \mu \mathrm{g} / \mathrm{mL}$ penicillin, $100 \mu \mathrm{g} / \mathrm{mL}$ streptomycin, $0.2 \%$ G5-supplement (ThermoScientific), $50 \mathrm{ng} / \mathrm{mL}$ nerve growth factor (NGF) $7 \mathrm{~s}$ (Alomone) and $10 \mu \mathrm{M}$ cytosine $\beta$-D-arabinofuranoside (Sigma-Aldrich), an antimitotic agent which reduces fibroblasts and glial cells enriching neurons in the primary cultures. Fifty per cent of the medium was refreshed every 3 days until day 9. At the 11th day of culture, fresh neurobasal-A medium supplemented only with $2 \%$ serum replacement (Sigma-Aldrich), $100 \mu \mathrm{g} / \mathrm{mL}$ penicillin and $100 \mu \mathrm{g} / \mathrm{mL}$ streptomycin was added to the myenteric ganglia culture. After 24 hours (12th day of culture), the supernatant (conditioned medium $(\mathrm{CM})$ ) was collected and stored at $-80^{\circ} \mathrm{C}$ until further use.

In vitro modulation of bone marrow-derived macrophages by myenteric ganglia conditioned medium

One day prior to the experiment, bone marrow-derived macrophages (BMDM; cultured as previously published ${ }^{17}$; see online supplementary materials) were seeded at $10^{6}$ cells $/ \mathrm{mL}$ in neurobasal-A medium containing $2 \%$ serum replacement, $100 \mu \mathrm{g} / \mathrm{mL}$ penicillin and $100 \mu \mathrm{g} / \mathrm{mL}$ streptomycin. The day of the experiment BMDM were incubated with CM collected from myenteric ganglia cultures or with control medium (neurobasal-A medium) 
containing $2 \%$ serum replacement, $100 \mu \mathrm{g} / \mathrm{mL}$ penicillin and $100 \mu \mathrm{g} / \mathrm{mL}$ streptomycin for 24 hours after which cells were rinsed with ice-cold dulbecco's phosphate buffered saline (D-PBS); Lonza) and collected for gene expression analysis.

In vitro modulation of M2-type bone marrow-derived macrophages For the polarisation of naïve BMDM (M0) into M2-type BMDM, we treated the naïve BMDM with $20 \mathrm{ng} / \mathrm{mL}$ interleukin (IL)4 (Peprotech) for 24 hours.

\section{Gene expression in sorted intestinal and bone marrow-derived macrophages and ganglia}

Total RNA was extracted from isolated ganglia, BMDM (M0 and M2); and sorted lamina propria macrophages (LPM $\phi)$ and $\mathrm{mM} \phi$ (see online supplementary materials) using the Rneasy Plus micro kit (Qiagen) and retrotranscribed by qScript cDNA SuperMix (Quanta Biosciences). The primers used are listed in online supplementary table 3 ). The expression levels of the genes of interest were normalised to the expression levels of the reference gene $r p l 32$.

\section{Preclinical model of POI}

Female mice aged 12-14 weeks were anaesthetised by intraperitoneal injection of a mixture of ketamine and xylazine. The anaesthetised mice underwent intestinal manipulation (IM) as previously described. ${ }^{18}$ Animals were subjected to either sham/placebo surgery (SHAM/PLAC), preoperative VNS, preoperative or postoperative administration of PRUC. For VNS, the right cervical vagus nerve was stimulated during $5 \mathrm{~min}(10 \mathrm{~Hz}, 1 \mathrm{~mA}$ and $1 \mathrm{~ms})$, while sham-operated mice only received a midline cervical incision. To evaluate the effect of PRUC, the mice received placebo, 1 or $5 \mathrm{mg} / \mathrm{kg}$ PRUC (Selleckchem) by oral gavage 1.5 hours prior to or 22.5 hours after the induction of POI. Vagotomy was performed 2 weeks prior to PRUC treatment via subdiaphragmatic transsection of both vagal trunks. To avoid gastric dilatation due to vagotomy, mice also underwent a pyloroplasty. ${ }^{12}$

\section{Human experiments}

For the randomised placebo-controlled pilot study, 42 patients were recruited at the University Hospital of Leuven between July 2014 and February 2016. Of these 42 patients, 30 completed the study and were analysed (figure 4B). Patients older than 18 years with confirmed or suspected neoplasms of the pancreas, ampulla Vateri or periampullary region undergoing an open pancreaticoduodenectomy were eligible. Patients were screened for the following exclusion criteria at the time of surgery: intra-abdominal inflammation, pregnancy, preoperative radiotherapy, chronic pancreatitis without suspected malignancy and uncontrolled diabetes $(200 \mathrm{mg} / \mathrm{dL})$. All patients were randomised in a 1:1:1 ratio to the SHAM/PLAC, VNS or PRUC group according to a computer-generated block randomisation list without stratification. The allocation sequence was generated by an independent pharmacist (University Hospital of Leuven) and was not available to the research team except for one clinical trial nurse (KV) operating the nerve stimulation during surgery. Placebo and PRUC packaging was identical and executed by the University Hospital of Leuven Pharmacy. After inclusion, the identity of the patients was encoded according to the European Union guidelines, that is, a unique number was used (hospital patient number) coupled with a unique case report form (CRF) number. Only the principal investigator had access to the list of participating subjects (hospital patient number) and the corresponding unique CRF number. The patients were treated with $2 \mathrm{mg}$ PRUC
(16 and 2 hours prior to surgery), abdominal VNS $(2 \mathrm{~min}, 20 \mathrm{~Hz}$, $2.5 \mathrm{~mA}, 1 \mathrm{~ms}$ at the start and end of the surgical procedure) or placebo (16 and 2 hours prior to surgery). Patients allocated to placebo or PRUC received sham stimulation at the start and end of surgery, patients treated with abdominal VNS received placebo. Abdominal VNS was performed as previously described (see online supplementary materials). ${ }^{11} 19$ An elaborate treatment protocol is also provided in online supplementary table 1 .

The primary end point was the reduction in surgery-induced upregulation of pro-inflammatory genes in duodenal muscularis tissue. In more detail, the local anti-inflammatory effect was determined in duodenal tissue taken at the start and 2 hours into the surgical procedure. As secondary end points, clinical recovery was assessed by the time of nasogastric tube (NGT) removal, volume of stomach output on postoperative day 3 , time to first solids, first defecation and time to discharge. The daily assessment of clinical recovery was performed by an experienced trial nurse. The nurses and treating physicians on the ward were not informed about the given treatment. The study was registered at clinicaltrials.gov (NCT02425774). All patients gave their written informed consent prior to their participation to the study.

\section{Statistics}

Normality, differences in variance and the presence of outliers were determined via the Shapiro-Wilk test, Levene test and Grubbs' test, respectively prior to statistical analysis. Two-way analysis of variance (ANOVA) followed by Bonferroni post hoc test was performed to compare multiple groups and multiple variables. To compare multiple groups and a single variable, one-way ANOVA or Kruskal-Wallis test was performed followed by Bonferroni or Dunn's post hoc test, respectively. To compare two independent groups and a single variable, the unpaired t-test or Mann-Whitney U test was performed depending on normality. To compare categorical variables the $\mathrm{X}^{2}$ test was used.

With respect to the pilot study, no previous data on changes in cytokine expression in human intestinal muscularis were available. Therefore, we arbitrarirly chose 10 patients per group to investigate the anti-inflammatory properties of VNS and preoperative administration of PRUC. Patients were excluded from the analysis of clinical recovery if they developed an abdominal infection (ie, positive culture of certain bacterial or fungal strains in abdominal drainage fluid) (SHAM/PLAC: $n=1$; VNS: $\mathrm{n}=3$ and PRUC: $\mathrm{n}=3$ ). This exclusion criteria was predefined based on previous publications ${ }^{20}$ showing that the occurrence of an abdominal infection is strongly positively correlated with prolonged postoperative gastroparesis and ileus. For the gene expression of the human muscularis tissue, one patient in the PRUC group was excluded, as this person was a severe outlier using the Grubb's outlier test $(\alpha=0.05)$. Probability level of $\mathrm{p}<0.05$ was considered statistically significant. Graphpad Prism software was used to perform statistical analysis.

\section{RESULTS}

\section{Enteric neurons dampen the activation of intestinal muscularis macrophages}

Previously, we reported neuromodulation of $\mathrm{mM} \phi$ in a murine model of POI, ${ }^{12}$ that is, VNS dampened the $\mathrm{mM} \phi$ activation and prevented intestinal manipulation-induced muscular inflammation. This effect is however indirect and most likely mediated via cholinergic enteric neurons, as not vagal terminals but enteric nerve fibres contact $\mathrm{mM} \phi .^{12}$ As shown in figure $1 \mathrm{~A}$, also in human, cholinergic enteric fibres are in close proximity to $\mathrm{mM} \phi$. To further prove this hypothesis, we evaluated to what extent electrical stimulation of enteric neurons indeed modulates $\mathrm{mM} \phi$ 

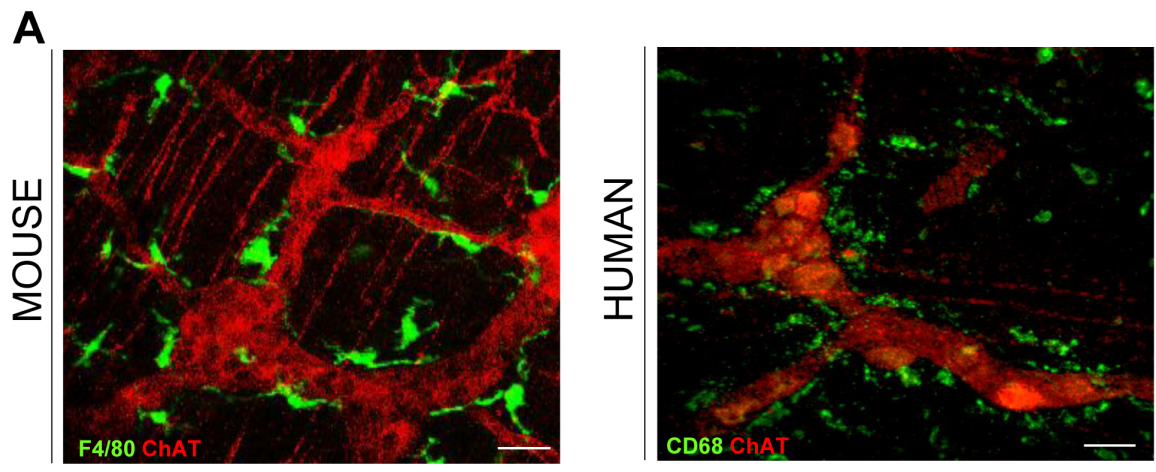

B
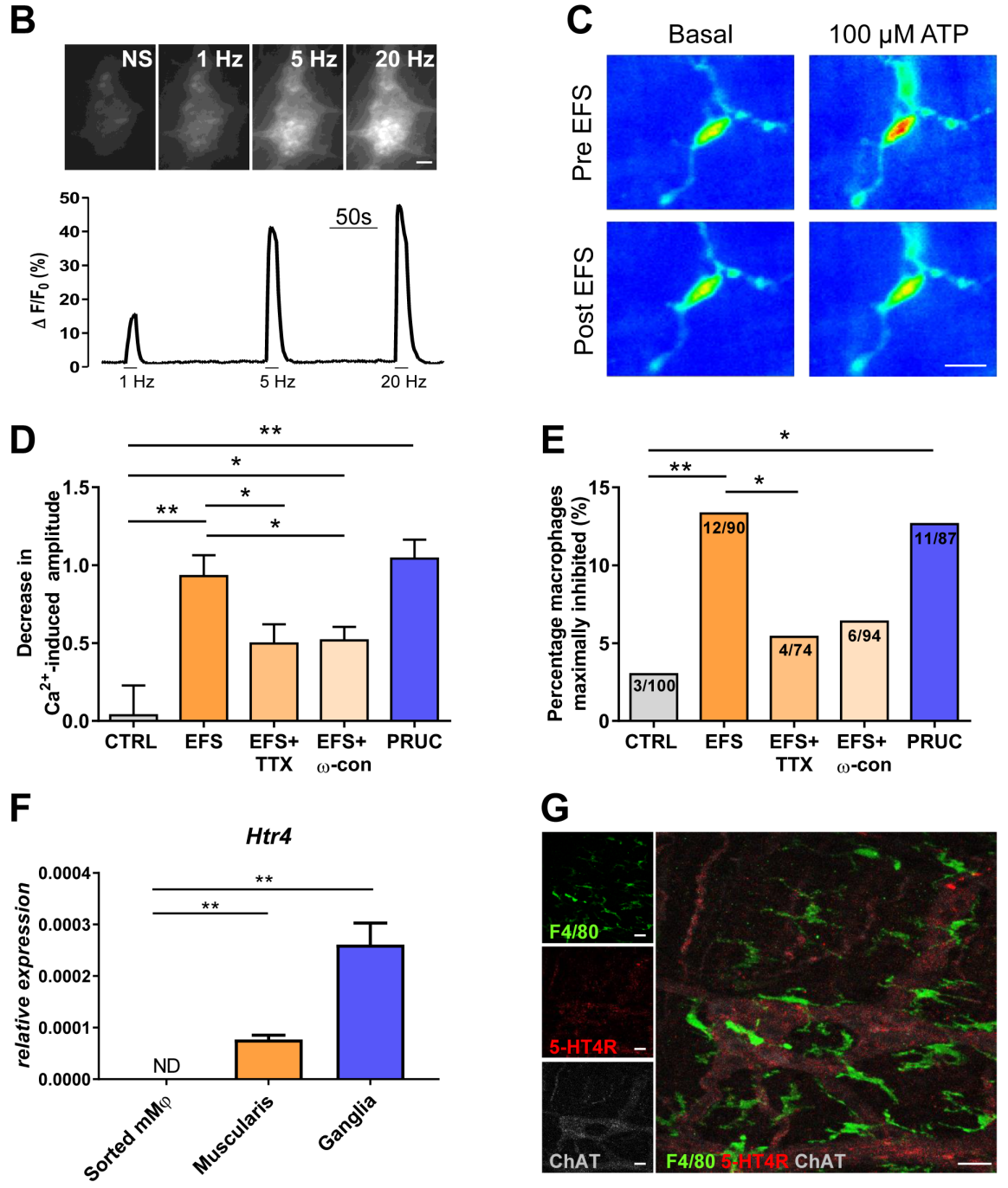

Figure 1 Cholinergic enteric neurons modulate resident muscularis macrophages. (A) Muscularis externa whole mount from the jejunal tissue of wild-type (WT) mice was stained for choline acetyltransferase (ChAT)-positive enteric neurons and F4/80-positive muscularis macrophages (mM $\phi$ ) (left panel). Muscularis externa whole mount from human small intestinal tissue was stained for CD68-positive mM $\phi$ and ChAT-positive enteric fibres (right panel). Scale bars are $50 \mu \mathrm{m}$. (B) Upper panel shows representative images and tracing of enteric neurons in the jejunal muscularis of Wnt. $1^{\text {GCAMP3 }}$ mouse before stimulation (no stimulation (NS)) and during electrical field stimulation with 1, 5 or $20 \mathrm{~Hz}$ (EFS; pulse width: 1 ms, current: $20 \mathrm{~mA}$ ). Scale bar is $10 \mu \mathrm{m}$. (C) Representative images from a Fluo-4 loaded mM $\phi$ in the jejunal muscularis of CX3CR $1^{\text {GFPMT }}$ mouse in response to $100 \mu \mathrm{M}$ of ATP before and after EFS ( $20 \mathrm{~Hz}$; pulse width: $1 \mathrm{~ms}$, current: $20 \mathrm{~mA})$. Scale bar is $20 \mu \mathrm{m}$. (D-E) Effect of Krebs (CTRL), EFS, EFS+tetradotoxin $(\mathrm{TTX} ; 10 \mu \mathrm{M}), \mathrm{EFS}+\omega$-conotoxin $(0.1 \mu \mathrm{M})$ or $150 \mu \mathrm{M}$ of prucalopride (PRUC) on ATP-induced mM $\phi$ activation, expressed as mean decrease in Ca ${ }^{2+}$ induced amplitude (D) and as percentage of $\mathrm{mM} \phi$ maximally inhibited (E) for $n=74-100 \mathrm{mM} \phi$. Data are from 4 to 7 animals/condition. ${ }^{*} P<0.05$, ${ }^{* *} p<0.01$. One-way analysis of variance with Bonferonni post hoc test (D) and two-tailed $\chi^{2}$ test (E). (F) 5 -HT4R expression in sorted mM $\phi$, isolated ganglia and whole mount muscularis tissue. (G) Maximum intensity projection of confocal stacks showing muscularis externa whole mount from the jejunum of WT mice was stained for 5-HT4R, ChAT and F4/80. 
activation. Using mice expressing the genetically encoded Cre-dependent $\mathrm{Ca}^{2+}$ sensor (GCaMP3) in enteric neurons (Wnt. $1^{\mathrm{GCaMP} 3}$ mice), we first confirmed frequency-dependent activation of enteric neurons by electrical field stimulation (EFS) in an in situ preparation of jejunal muscularis tissue (figure 1B). Next, we studied the effect of EFS on $\mathrm{mM} \phi$ stimulated with ATP, a typical tissue damage-associated molecule. EFS significantly reduced the amplitude of the ATP-induced $\mathrm{Ca}^{2+}$ signal and increased the number of maximally inhibited $\mathrm{mM} \phi$ (figure $1 \mathrm{C}-\mathrm{E}$ ). Tetrodotoxin, a compound blocking the generation of action potentials in neurons, reduced the inhibitory effect of EFS (figure 1D-E). Similar effects were obtained using $\omega$-conotoxin, a blocker of voltage-gated $\mathrm{Ca}^{2+}$ channels, predominantly expressed in nerve terminals, where they control neurotransmitter release, ${ }^{21}$ further supporting the neuronal origin of the inhibitory effect of EFS (figure 1D-E).

Next, we evaluated the effect of the selective 5-HT4R agonist PRUC on $\mathrm{mM} \phi$ activation in whole mount muscularis preparations. As shown in figure 1D,E, incubation with PRUC mimicked the effect of EFS. These data suggest that neurotransmitters released by enteric neurons modulate $\mathrm{mM} \phi$ activity. Of note, although we applied a higher dosing of PRUC in these in situ experiments than previously described in literature, ${ }^{15} 22$ this concentration did not affect pro-inflammatory and anti-inflammatory cytokine expression in lipopolysaccharide (LPS)-stimulated BMDM (data not shown), argueing against a direct effect of PRUC on M $\phi$. To further confirm that PRUC is truly acting on the enteric neurons, and not directly interacts with $\mathrm{mM} \phi$, the expression of 5-HT4R was assessed in sorted $\mathrm{mM} \phi$, isolated enteric ganglia and muscularis tissue using real-time quantitative PCR. 5-HT4R expression was not detected in sorted $\mathrm{mM} \phi$, but was abundantly present in isolated enteric ganglia and muscularis tissue (figure 1F). This was confirmed in immunohistochemical stainings of muscularis tissue. Submucosal and myenteric neurons were $5 \mathrm{HT} 4 \mathrm{R}$ positive, but not the $\mathrm{mM} \phi$. Interestingly, F4/80-positive $\mathrm{mM} \phi$ were found in close proximity to cholinergic enteric fibres expressing 5HT4R (figure $1 \mathrm{G}$ ). To further exclude a direct effect of PRUC on macrophages $(\mathrm{M} \phi)$, LPS-stimulated whole blood of healthy volunteers was pretreated with PRUC. No effect on IL6, IL8 or tumour necrosis factor (TNF)- $\alpha$ release was observed (see online supplementary figure 1), further excluding this possibility.

\section{Prucalopride administered prior to but not after surgery reduces intestinal inflammation in vivo and improves POI}

Although cervical VNS effectively reduces intestinal inflammation and shortens POI in preclinical models, ${ }^{10} 1223$ this approach is invasive and stimulation parameters in human still require optimisation. Moreover, vagal innervation declines in density along the intestinal tract and fails to innervate the distal colon, making this approach less attractive for clinical application. As we showed that PRUC dampens $\mathrm{mM} \phi$ activation, we asked whether it can mimic the effect of VNS in vivo and subsequently prevent POI. As with VNS, PRUC was administered prior to surgery in order to precondition $\mathrm{mM} \phi$. As shown in figure 2, preoperative administration of $5 \mathrm{mg} / \mathrm{kg}$ PRUC, similar to VNS, reduced intestinal inflammation, that is, cytokine expression and influx of inflammatory cells (see online supplementary figure 2, figure 2A-E). Moreover, intestinal transit assessed 24 hours after surgery was restored in mice pretreated with PRUC or VNS compared with SHAM/PLAC stimulation (figure 2B). Vagotomy did not affect the anti-inflammatory effects of PRUC (figure 2F-G), indicating that PRUC acts directly on the intestine. Notably, PRUC, administered 24 hours after surgery did not reduce intestinal inflammation and failed to restore intestinal transit (figure 2A-E). Taken together, these data indicate that PRUC has anti-inflammatory properties, but only when administered before surgery, and effectively reduces POI.

\section{The anti-inflammatory effect of prucapride is mediated by $\alpha 7 n A C h R$}

As we previously demonstrated that the anti-inflammatory effect of VNS is mediated by $\alpha 7 \mathrm{nAChR},{ }^{12}{ }^{23}$ we next evaluated its role in the effect of PRUC. First, we determined the expression of $\alpha 7 \mathrm{nAChR}$ using immunohistochemical stainings. As shown in figure $3 \mathrm{~A}, \alpha 7 \mathrm{nAChR}$ was expressed in both murine and human intestine. To confirm this finding, expression of $\alpha 7 \mathrm{nAChR}$ in sorted $\mathrm{mM} \phi$ was compared with that of isolated lamina propria $\mathrm{M} \phi$. As shown in figure $3 \mathrm{~B}, \alpha 7 \mathrm{nAChR}$ was only expressed in isolated $\mathrm{mM} \phi$.

As $\mathrm{mM} \phi$ were reported to have a M2-like tolerogenic phenotype ${ }^{24}$ we next evaluated to what extent enteric neurons contribute to this phenotype and instal the expression of $\alpha 7 \mathrm{nAChR}$. To this end, BMDM were incubated with conditioned medium of primary myenteric ganglia cultures. As shown in figure $3 \mathrm{C}-\mathrm{D}$, conditioned medium induced the expression of $\alpha 7 \mathrm{nAChR}$ and installed a M2-like phenotype, as indicated by the upregulation of Arg1, Retnla, Cd163, Il10 and Cx3cr1. Of note, also IL4-differentiated BMDM expressed $\alpha 7 \mathrm{nAChR}$, suggesting that M2-like tissue $\mathrm{M} \phi$ may be under neuronal modulation (figure 3E). Finally, we performed western blot analysis of murine intestinal immune cells lysates with affinity purification on an $\alpha$-bungarotoxin ( $\alpha$-Bgtx)-sepharose column (figure $3 \mathrm{~F}$ ). No $\alpha 7 \mathrm{nAChR}$ signal was observed in the CD $45^{+}$ population devoid of $\mathrm{mM} \phi$, while three bands were detected in the $\alpha$-Bgtx purified $\mathrm{mM} \phi$ lysate of wild-type mice. Of interest, the molecular weight differed from the $\alpha 7 \mathrm{nAChR}$ isolated from the brain and the predicted $56 \mathrm{kDa}^{2526}$ The bands detected were absent in $\alpha 7 \mathrm{nAChR} \mathrm{KO}$ mice, confirming the specificity of the antibody used. To the best of our knowledge, this is the first specific western blot analysis of $\mathrm{mM} \phi-\alpha 7 \mathrm{nAChR}$, suggesting that the $\alpha 7 \mathrm{nAChR}$ in $\mathrm{mM} \phi$ differs from its neuronal counterpart, although proteolysis during the purification cannot be excluded.

Next, we studied the effect of PRUC in our POI model using $\alpha 7$ nAChR KO mice. Of note, PRUC failed to improve intestinal transit (figure $3 \mathrm{G}$ ) and did not reduce the number of myeloperoxidase (MPO)-positive cells (figure $3 \mathrm{H}$ ), or Il6 and Il $1 \alpha$ expression (figure $3 \mathrm{I}-\mathrm{J}$ ) in the muscularis. Taken together, these data indicate that the anti-inflammatory effect of PRUC, similar to VNS, is mediated by $\alpha 7 \mathrm{nAChRs}$.

\section{Anti-inflammatory effect of preoperative administration of prucalopride in humans}

We next aimed to translate our preclinical data to patient care. Therefore, we designed a placebo-controlled pilot study comparing PRUC $(n=10)$, abdominal VNS $(n=10)$ and SHAM/ PLAC treatment $(n=10)$ in patients undergoing a pancreaticoduodenectomy (figure 4A-B). The demographic and clinical characteristics of all groups were similar except for male gender, which was higher in the PRUC group (see online supplementary table 2). Previous studies, however, demonstrated that gender did not have a clinical relevance on postoperative outcome. ${ }^{2027} 28$ The current patient population was selected based on high incidence of POI and the long duration of the surgical procedure allowing us to detect changes in pro-inflammatory gene expression in the muscularis. Indeed, 2 hours after the start of the 
A
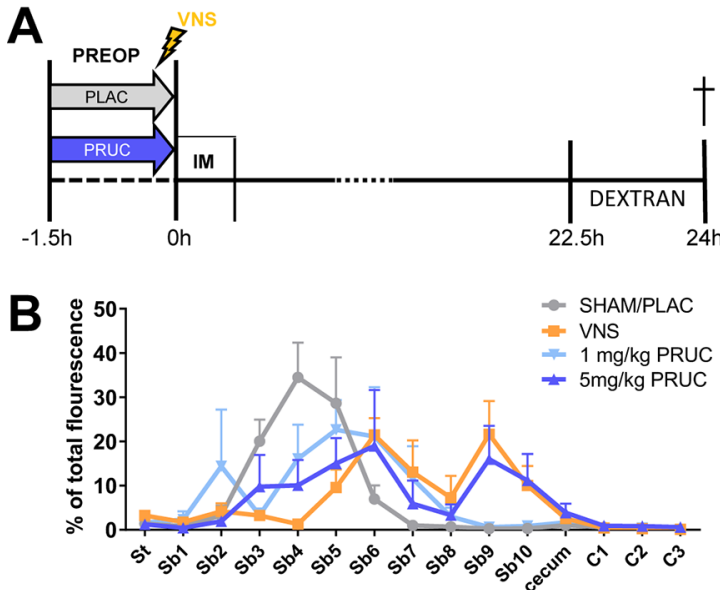

C
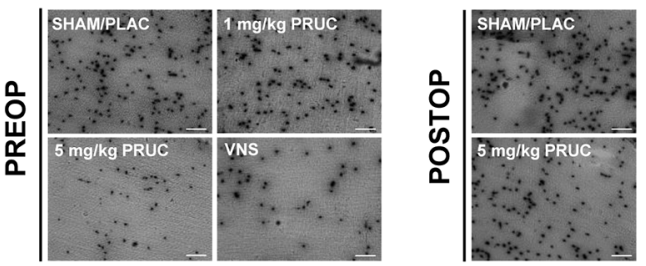
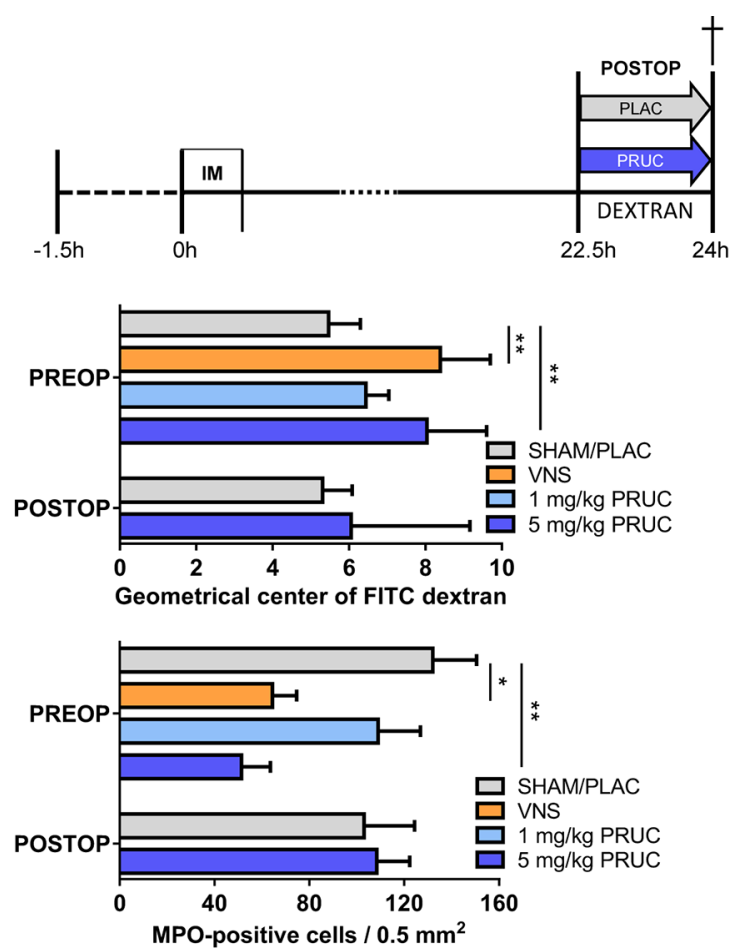

MPO-positive cells $/ 0.5 \mathrm{~mm}^{2}$


Figure 2 Pharmacological activation of enteric neurons reduces intestinal inflammation and improves postoperative ileus. (A) Schematic representation of experimental protocol. Mice subjected to intestinal manipulation (IM) were treated with vagus nerve stimulation (VNS) or prucalopride (PRUC; oral gavage 1.5 hour prior to or 22.5 hours after IM) and compared to sham/placebo (SHAM/PLAC) . Fluorescently labelled dextran was gavaged 22.5 hours after IM and mice were sacrificed 1.5 hours later. Geometrical centre (GC) of dextran distribution was used to quantify the GI transit. (B) Dextran distribution through the GI tract (left panel) and GC (right panel) for each group of mice. St, stomach; $\mathrm{Sb}$, small bowel; C, colon. (C) Representative image (left panel) and number of myeloperoxidase (MPO)-positive cells/0.5 $\mathrm{mm}^{2}$ (right panel) in jejunal muscularis. Scale bars are $50 \mu \mathrm{m}$. (D-E) Relative mRNA levels for $/ 16$ and $/ 11 \alpha$ normalised to the housekeeping gene rp/32 from jejunal muscularis tissue. (B-E) Data are expressed as mean \pm SEM from $n=6-10$ mice/group. ${ }^{*} \mathrm{P}<0.05$, ${ }^{* *} \mathrm{p}<0.01$. One-way ANOVA with Bonferonni post hoc test for PREOP and unpaired t-test for POSTOP. (F-G) Vagotomised (VGX) mice treated with PLAC or PRUC 1.5 hours prior to IM. (F) Bar graph represents the mean GC 24 hours after IM. (G) Mean of relative mRNA levels in the jejunal muscularis for $/ 11 \alpha$ and $/ 16$ normalised to the housekeeping gene rp/32. ${ }^{*} \mathrm{P}<0.05,{ }^{*} \mathrm{p}<0.01$. Unpaired t-test for VGX-PLAC vs VGX-PRUC. $n=6-8$ mice/group.

surgical procedure, the pro-inflammatory genes $I l 6, I l 8$ and Ccl2 were significantly upregulated in the intestinal muscularis tissue (figure 5A). Of note, PRUC significantly reduced $I l 6$ and Il8 expression in the muscularis, while VNS tended to reduce Il6 compared with SHAM/PLAC treatment. The serum levels of IL6, IL8 and TNF- $\alpha$ were not affected by PRUC or VNS (see online supplementary figure 3 ).

\section{Preoperative administration of prucalopride improves clinical recovery in humans}

As shown in figure 5B-C PRUC, but not VNS, significantly reduced the time to removal of the NGT, NGT output and time to first solids. In more detail, there was a wide spread in the aforementioned clinical parameters in the SHAM/PLAC-treated group. In contrast, the NGT output was below $350 \mathrm{~mL}$ at day 3 in all PRUC-treated patients as opposed to 3 and 6 in the SHAM/ PLAC-treated group and VNS-treated group, respectively. In line, the NGT was removed within 4 days in all PRUC-treated patients compared with three patients in the SHAM/PLAC group and three in the VNS group. In line, all PRUC-treated patients tolerated solid foods by day 5 in contrast to only two and one patient in the SHAM/PLAC group and VNS group, respectively. No difference in time to first defecation was observed between the three different groups. Of note, all PRUC-treated patients tolerated solids and had their first defecation by day 9 , as opposed to only five and two patients in the SHAM/PLAC group and VNS group, respectively. Finally, time to discharge was significantly reduced from $15.0(12.5-20.5)$ days in the SHAM/PLAC group to 10.5 (8.0-15.5) days in the PRUC group (figure 5C).

Taken together, our data show that preoperative administration of PRUC has anti-inflammatory properties in human, as shown by the downregulation of pro-inflammatory cytokines in the intestinal muscularis, and leads to a faster postoperative clinical recovery. 
A



B

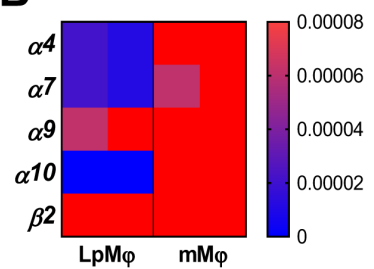

E

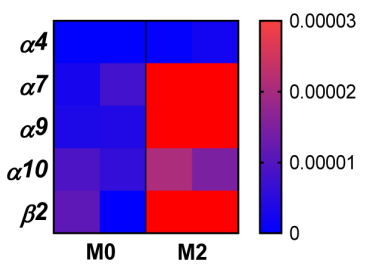

H

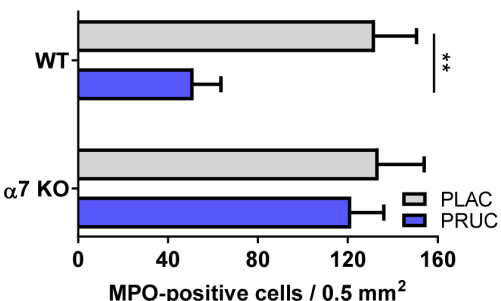

MOUSE

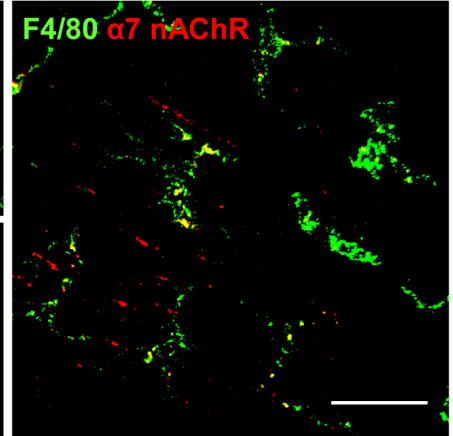

C

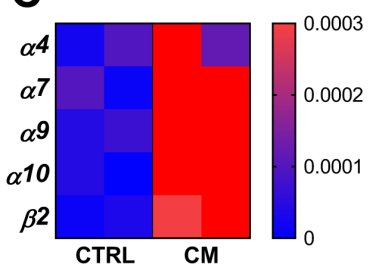

CTRL

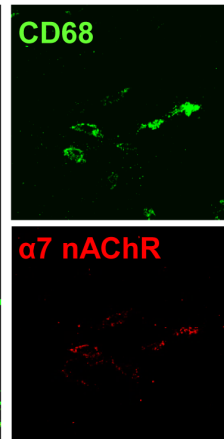

D
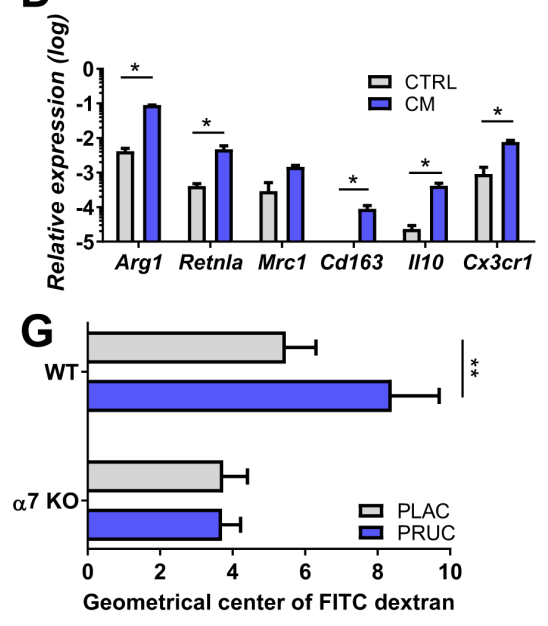

J

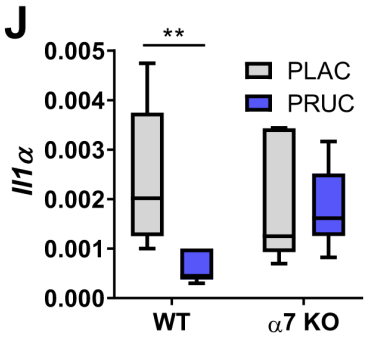

Figure 3 Cholinergic modulation of muscularis macrophages $(\mathrm{mM} \phi)$ is mediated by alpha7 nicotinic acetylcholine receptor $(\alpha 7 \mathrm{nAChR})$. (A) Maximum intensity projection of confocal stacks showing small intestinal muscularis whole mount tissue stained for $\alpha 7 \mathrm{nAChR}$ (red) on F4/80-positive muscularis macrophages (mM $\phi$; green) in a wild-type (WT) mouse (right panel) and on CD68-positive mM $\phi$ (green) in human (left panel). Scale bars are $20 \mu \mathrm{m}$. (B) Heatmap of nAChR subunit expression in sorted lamina propria macrophages (LpM $\phi)$ and $\mathrm{mM} \phi$. (C) Heatmap of nAChR subunit expression in naïve bone marrow-derived macrophages (BMDM) incubated with conditioned medium (CM) collected from myenteric ganglia cultures or control medium (CTRL). (D) Bar graph of anti-inflammatory gene expression of naïve BMDM incubated with CM collected from myenteric ganglia cultures or CTRL. Data are expressed as mean $\log 10 \pm S E M$. ${ }^{*} \mathrm{P}<0.05$. Unpaired t-test. $n=3-5 /$ experiment. (E) Heatmap of $n A C h R$ subunit expression in BMDM (M0) and polarised M2 BMDM. (F) Western blot analysis of sorted mM $\phi$ and sorted muscularis immune cells (CD45 ${ }^{+}$CD11 $\mathrm{c}^{-} \mathrm{IA} / \mathrm{IE} \mathrm{E}^{-} \mathrm{CD} 64^{-} \mathrm{CD} 11 \mathrm{~b}^{-}$)

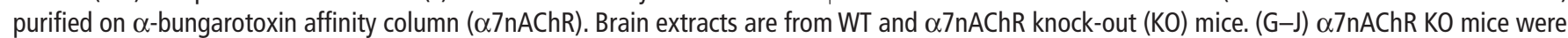
treated with either placebo (PLAC) or prucalopride (PRUC) 1.5 hours prior to intestinal manipulation (IM). (G) Bar graph represents the GC values for each group 24 hours after IM. (H) Bar graph represents the number of MPO-positive cells $/ 0.5 \mathrm{~mm}^{2} 24$ hours after IM. (G-H) Data are expressed as mean \pm SEM. ${ }^{*} \mathrm{P}<0.01$. Unpaired t-test. $\mathrm{n}=6-8$ mice/group. (I-J) Relative mRNA levels in jejunal muscularis for $/ / 1 \alpha$ and $/ / 6$ normalised to the housekeeping gene rp/32. Data are expressed as median with 5-95 percentile whiskers. ${ }^{*} \mathrm{P}<0.01$. Mann-Whitney U test. $\mathrm{n}=6-8 \mathrm{mice} / \mathrm{group}$.

\section{DISCUSSION}

In the present study, we provide in vitro evidence that enteric neurons, either stimulated electrically or via administration of PRUC, can dampen $\mathrm{mM} \phi$ activation. In vivo, PRUC administered prior to, but not after surgery mimicked the effect of VNS in a murine model of POI. Finally, in a pilot study, we showed that also in human, administration of PRUC prior to a Whipple procedure has an anti-inflammatory effect, and improves clinical postoperative parameters. Collectively, these data provide further evidence supporting the concept that enteric neuron potentation exerts an inhibitory input to $\mathrm{mM} \phi$ and indicate that preoperative activation of this pathway by PRUC, or other 5-HT4R agonists, should be further explored as treatment of POI.

The intestine is densely populated with enteric neurons, organised into the myenteric and submucosal plexus, mediating a variety of physiological processes such as intestinal motility, 
A

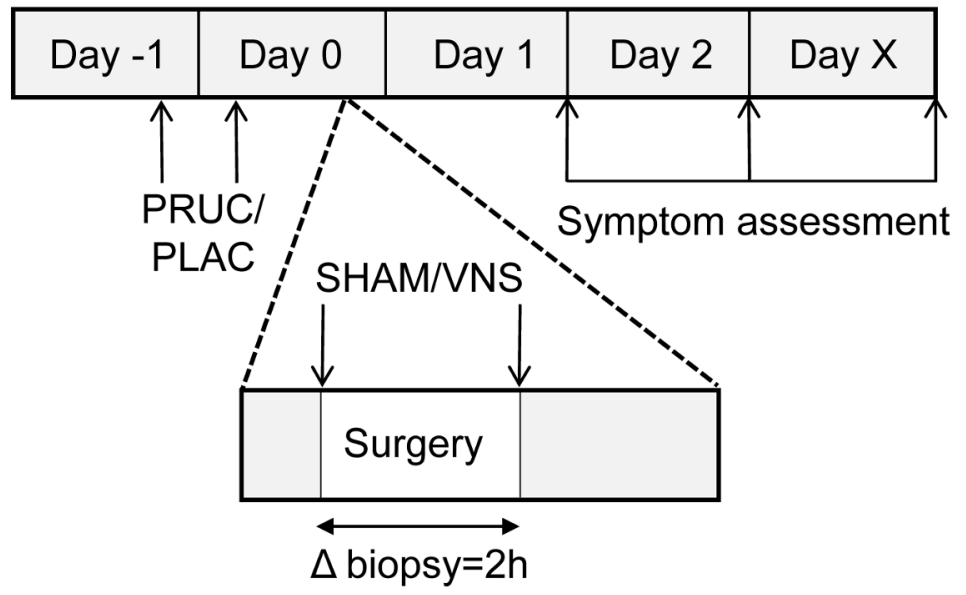

Figure 4 (A) Patient flow chart. (B) Schematic overview of study protocol. PLAC, placebo; PRUC, prucalopride; VNS, vagus nerve stimulation.

secretion and control of blood flow. Only recently, it has become clear that myenteric neurons are critically important for the maintenance of $\mathrm{mM} \phi$ by secretion of colony stimulatory factor 1 , a growth factor required for $\mathrm{mM} \phi$ development. ${ }^{24}{ }^{29}$ Here, we provide further evidence that enteric neurons communicate with $\mathrm{mM} \phi$ and can dampen their activation in response to ATP. Using an experimental set-up in which $\mathrm{mM} \phi$ can be studied in their natural environment, we showed that electrical stimulation of enteric neurons reduced the ATP-induced $\mathrm{Ca}^{2+}$ response in $\mathrm{mM} \phi$, previously shown to be coupled to cytokine secretion. ${ }^{30}$ This effect was dampened by the neurotoxins tetrodotoxin and $\omega$-conotoxin, indicating that the reduction in the $\mathrm{mM} \phi$ response to ATP resulted from neuronal activation. Although the exact nature of the neurons involved was not identified, our previous work demonstrating that 1 ) the anti-inflammatory effect of VNS is mediated by $\alpha 7 \mathrm{nAChR}, 2$ ) both nicotine and the $\alpha 7 \mathrm{nAChR}$ agonist choline also dampen $\mathrm{mM} \phi$ activation by ATP and 3) cholinergic fibres are in close contact with $\mathrm{mM} \phi$, are all indicative that $\mathrm{mM} \phi$ are modulated by cholinergic neurons via $\alpha 7 \mathrm{nAChR} .{ }^{12}$ Of interest, $\alpha 7 \mathrm{nAChR}$ expression was restricted to $\mathrm{mM} \phi$ and $\mathrm{M} 2$-type BMDM and could be induced by conditioned medium of myenteric ganglia, further providing evidence that enteric neurons communicate with $\mathrm{mM} \phi$ and modulate their function. Although identification of the neuronal mediators involved requires further study, one can speculate that this process may be crucial to facilitate the inhibitory input of $\mathrm{ACh}$ 


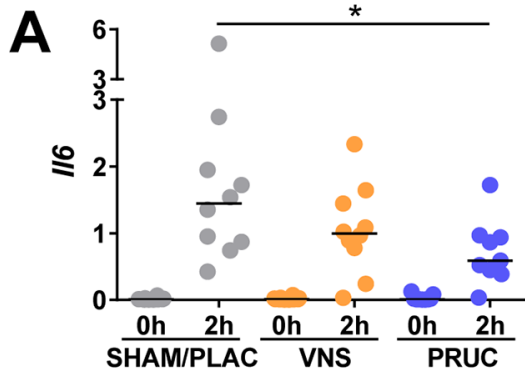

B
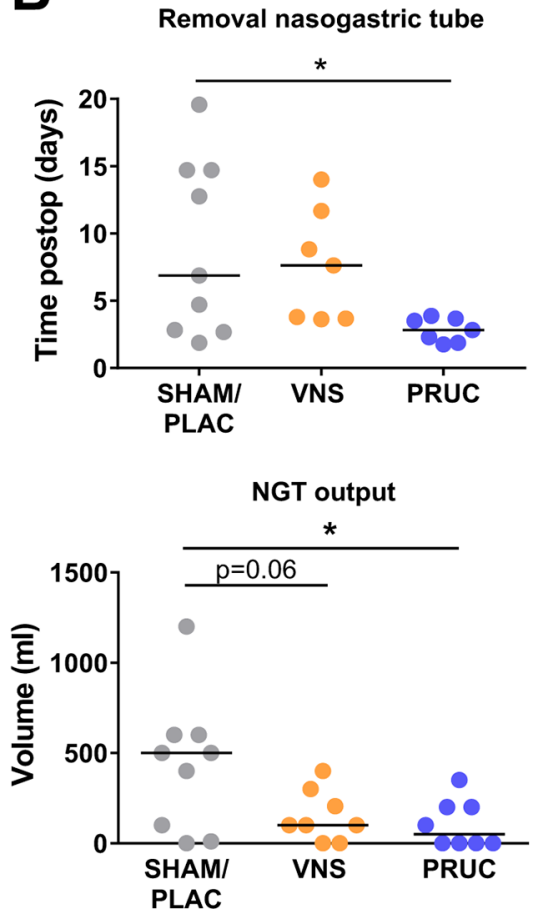

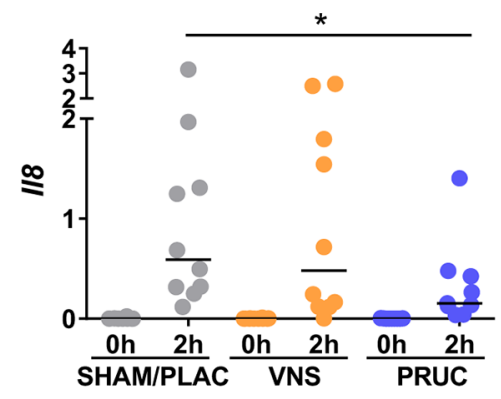

C

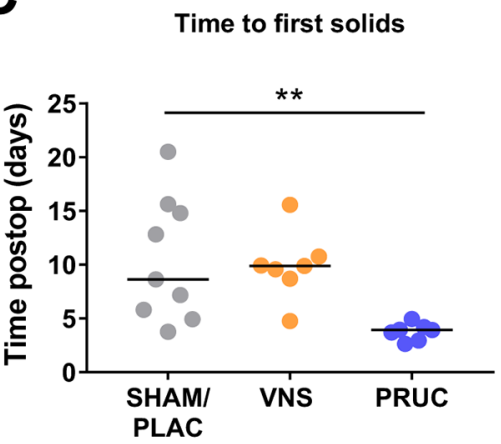

First solids and defecation

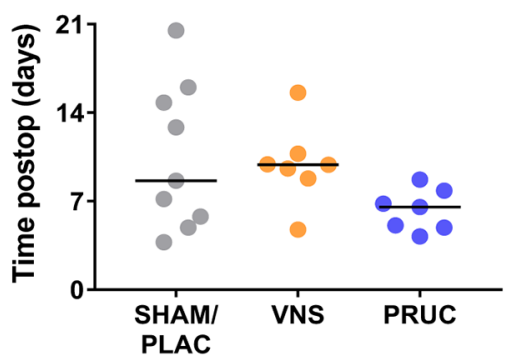

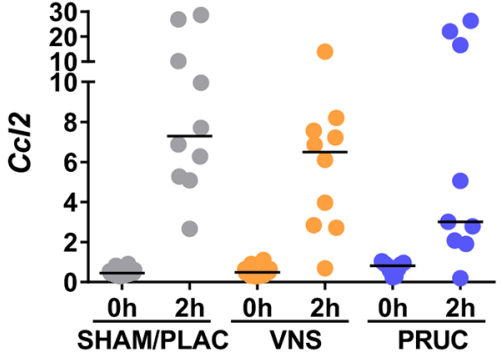

Defecation

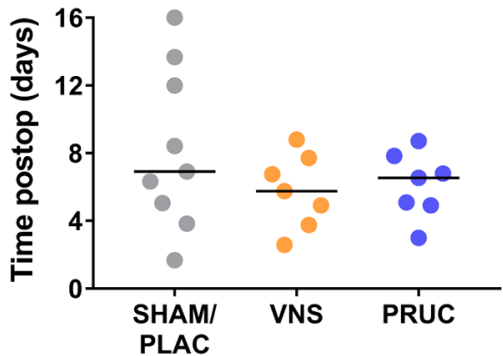

Time to discharge

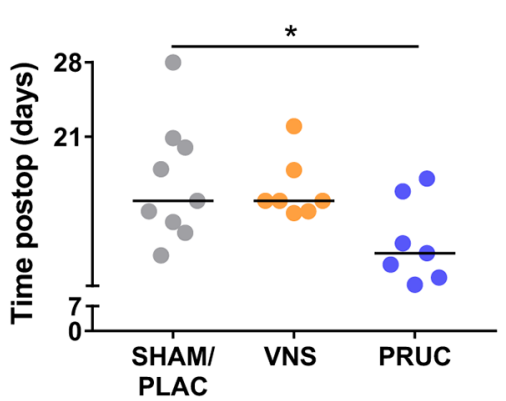

Figure 5 Preoperative administration of prucalopride reduces intestinal inflammation and improves postoperative ileus in human. (A) Relative mRNA levels in the muscularis externa for $1 / 6,1 / 8$ and $C \mathrm{C} / 2$ normalised to the housekeeping gene 10 fru3. Duodenal tissue was taken at the beginning of the procedure ( 0 hour) and 2 hours into the surgery ( 2 hours) for patients treated with sham/placebo (SHAM/PLAC), abdominal vagus nerve stimulation (VNS) or prucalopride (PRUC). Data are expressed as mean $\pm S E M{ }^{*} P<0.05$. Repeated two-way analysis of variance (ANOVA) with Bonferroni correction for multiple testing; $n=9-10$ patients/group. (B) Time until removal of nasogastric tube (NGT) (upper panel) and the NGT output on postoperative day 3 (lower panel). (C) Other clinical (secondary) end points (ie, days until tolerance to first solids, first defecation, combination of time to tolerance to solids and first defecation and days until discharge) for SHAM/PLAC-treated, abdominal VNS-treated or PRUC-treated groups without site-specific complications (SSC). (C) NGT output data on postoperative day 3 is expressed as median \pm interquartile range for SHAM/PLACtreated, abdominal VNS-treated or PRUC-treated groups without SSC. ${ }^{*} \mathrm{P}<0.05$. Kruskal-Wallis test with Dunn's test for multiple comparison test; $\mathrm{n}=7-9$ patients without SSC/group. (B-C) The other dot plots are expressed as mean $\pm S E M{ }^{*} \mathrm{P}<0.05$, * $\mathrm{p}<0.01$. One-way ANOVA with Bonferroni correction for multiple testing; $\mathrm{n}=7-9$ patients without SSC/group.

to $\mathrm{mM} \phi$. The ' $\mathrm{mM} \phi-\alpha 7 \mathrm{nAChR}$ ' by which this inhibitory effect is mediated seems to differ from the 'classical neuronal $\alpha 7 \mathrm{nAChR}$ ', as shown by the different molecular weight.

In previous studies, we demonstrated that VNS prior to abdominal surgery prevents intestinal inflammation and improves POI in a murine model, an effect mediated by close interaction of the vagus nerve with enteric neurons. ${ }^{11-13}$ The clinical application of VNS to treat POI is however not ideal as the vagal innervation declines along the GI tract and is even absent in the distal colon. ${ }^{31}$ An alternative would be to pharmacologically enhance ACh release by enteric neurons along the GI tract. Of interest, the 5-HT4R agonist PRUC is acknowledged as a panenteric prokinetic shown to be effective by enhancing the release of ACh. ${ }^{14}{ }^{32}$ In the present study, we show that in vivo, a single administration of PRUC prior to surgery reduced the upregulation of the pro-inflammatory cytokines, prevented the influx of inflammatory cells and improved intestinal transit in mice. As 5-HT4R was solely expressed by enteric neurons, but not on $\mathrm{mM} \phi$, and pretreatment of PRUC could not reduce the pro-inflammatory cytokine release in LPS-stimulated whole blood, these data indicate that PRUC exerts its beneficial effect via interaction with enteric neurons and not through a direct effect on M $\phi$. Similar to VNS, the therapeutic effect of PRUC was mediated by $\alpha 7 \mathrm{nAChR}$, as it was absent in $\alpha 7 \mathrm{nAChR} \mathrm{KO}$ mice. These data are in line with a previous report in rats showing that the 5-HT4R agonist mosapride improved POI and reduced intestinal inflammation, an effect that was attenuated by the nicotinic antagonist hexamethonium and the $\alpha 7 \mathrm{nAChR}$ antagonist methyl lycaconitine citrate. ${ }^{33}$ Moreover, as vagotomised mice still improved with PRUC, we can preclude the possibility 
that the anti-inflammatory effect of PRUC was mediated by the vagus nerve. Our data thus indicate that PRUC prevents the influx of monocytes and neutrophils via 5-HT4R activation of enteric neurons. ${ }^{33}$

Another important finding of our study is the observation that administration of PRUC after surgery is devoid of an anti-inflammatory effect and fails to restore GI transit. Indeed, PRUC administered 22.5 hours after surgery had no significant effect on intestinal inflammation and transit. These data would argue against the use of prokinetics to improve intestinal motility in the postoperative phase, especially as the contractile potential of the inflamed intestine is compromised. In previous clinical trials, prokinetics have always been administered in the postoperative phase with rather disappointing results. Erythromycin, metoclopramide, cisapride ${ }^{3}$ or ghrelin receptor agonists ${ }^{4}$ all failed to improve POI. Two studies with mosapride started on 1 day after surgery showed reduction in time to first defecation and improvement of solid food intake, but these trials were small $(n=30-40)$ or single blinded..$^{34} 35$ Of particular interest in view of our findings, two studies have evaluated the effect of PRUC in patients undergoing colorectal surgery, showing no effect on hospital stay ${ }^{36}$ or a reduction from 8 to $7 .{ }^{37}$ In both studies, treatment was continued for 3-7 days but was only started the day after surgery. Based on our preclinical data, one would argue that patients should be pretreated with PRUC prior to surgery in order to dampen the inflammatory response in the muscularis evoked by abdominal surgery.

To evaluate this novel approach in human, we designed a pilot study in patients undergoing a Whipple procedure. This surgical procedure was chosen in view of its long duration allowing the detection of upregulation of inflammatory genes, such as Il6, Il8 and Ccl2, as previously reported. ${ }^{7}$ Although we recently reported a reduction in IL8 and IL6 production by whole blood in patients who underwent abdominal VNS, ${ }^{11}$ no effect was noted on gene expression in the muscularis externa of the VNS group. In contrast, expression of both $I l 6$ and $I l 8$ was significantly reduced in the PRUC-treated patients, compared with SHAM/PLAC treatment. With only 2 times $2 \mathrm{mg}$ of PRUC administered prior to surgery, clinical parameters were significantly better in the PRUC group. Nasogastric output and its time of removal and time to tolerance of solid food were significantly reduced compared with the other groups. Although no strict criteria were defined a priori to discharge patients, hospital stay was significantly shorter (approximately 5 days) in the PRUCtreated group compared with the SHAM/PLAC-treated group. To what extent the clinical parameters assessed in our pilot study would have been even more improved if PRUC treatment was continued after surgery remains to be studied. Obviously, as our clinical study is only a pilot study and not powered for clinical parameters, these data should be interpreted with care. Nevertheless, together with the preclinical data, our study indicates that preoperative administration of PRUC, and perhaps also other 5-HT4R agonists, dampens the inflammatory response triggered by abdominal surgery, and leads to faster clinical recovery. Besides this protective, anti-inflammatory effect of PRUC, activation of 5-HT4R also possesses neuroprotective properties in the intestine, both in vitro and in vivo,${ }^{38-41}$ further underscoring the therapeutic potential of 5-HT4R agonists in the treatment of POI.

Based on the data provided, pharmacological management of POI should be changed considerably, that is, clinicians should reconsider administering prokinetics only in the postoperative period in an attempt to stimulate gut motility. Instead, patients should be treated with 5-HT4R agonists like PRUC prior to surgery to prevent $\mathrm{mM} \phi$ activation and intestinal inflammation. Our study sets the stage for future clinical trials evaluating the effect of 5-HT4R agonists starting prior to surgery.

\section{Author affiliations}

${ }^{1}$ Department of Chronic Diseases, Metabolism and Ageing, Translational Research Center for Gastrolntestinal Disorders, Intestinal Neuroimmune Interactions, University of Leuven, Leuven, Belgium

${ }^{2}$ Department of Abdominal Surgery, University Hospital of Leuven, Leuven, Belgium ${ }^{3}$ Department of Chronic Diseases, Metabolism and Ageing, Translational Research Center for Gastrointestinal Disorders, Laboratory for Mucosal Immunology, University of Leuven, Leuven, Belgium

${ }^{4}$ Department of Cellular and Molecular Medicine, Laboratory of Ion Channel Research, KU Leuven; VIB Center for Brain \& Disease Research, Leuven, Belgium ${ }^{5}$ Department of Anesthesiology, University Hospital of Leuven, Leuven, Belgium ${ }^{6}$ Department of Cellular and Molecular Medicine, Laboratory of Protein Phosphorylation and Proteomics, Universitiy of Leuven, Leuven, Belgium ${ }^{7}$ CNR, Neuroscience Institute-Milano, Biometra University of Milan, Milan, Italy ${ }^{8}$ Department of Pharmaceutical and Pharmacological Sciences, Laboratory for Drug Delivery and Disposition, University of Leuven, Leuven, Belgium

${ }^{9}$ Department of Chronic Diseases, Metabolism and Ageing, Translational Research Center for Gastrointestinal Disorders, Laboratory for Enteric Neuroscience, University of Leuven, Leuven, Belgium

Acknowledgements The authors would also like to thank R Mols, I Terrasson, B Topal and the nursing staff (Oka1, E447 and E906) of UZ Leuven for their excellent technical assistance. Confocal imaging (LSM880: AKUL/15/37 - G0H1816N to PVDB) and calcium imaging were performed at the Cell Imaging Core (KU Leuven, Belgium). Cell sorting and flow cytometry was done at the FACS core facility (KU Leuven, Belgium).

Contributors NS, EL, PJG-P, KT, GM and GEB planned and designed experiments. NS, EL, PJG-P, GF, IA, EM, MS, MFV, GB, EG-D, YAA, PA, SD, GG, EW, RD, AW, RA, $A D H, K V, S V, M M, C G, M V, K T$ and PVDB performed or supervised the experiments. NS, EL, PJGP, GM and GEB reviewed data and wrote the manuscript. All other authors corrected and approved the final version of the manuscript.

Funding This work was supported by the European Research Council (ERC) Advanced Grant (ERC-2013-Adg: 340101 Cholstim) to GEB. GEB is also supported by Flanders Fund for Innovation by Science and Technology (IWT-TBM; 110699), and Research Foundation-Flanders (FWO): Odysseus programme (G.0905.07) and two FWO grants (G.0566.12N and G.0890.18N). NS, PJG-P, YAA, GG, SV and GM are supported by a postdoctoral research fellowship of FWO. GM is supported by an FWO grant (G.0D83.17N) and by KU Leuven grants (ZKD2906-C14/17/097 and ZKC9531-C12/15/016).

Competing interests None declared.

Patient consent Obtained.

Ethics approval This study was approved by KU Leuven Animal Ethics Committee and Medical Ethics Committee UZ KU Leuven.

Provenance and peer review Not commissioned; externally peer reviewed.

Open access This is an open access article distributed in accordance with the Creative Commons Attribution Non Commercial (CC BY-NC 4.0) license, which permits others to distribute, remix, adapt, build upon this work non-commercially, and license their derivative works on different terms, provided the original work is properly cited, appropriate credit is given, any changes made indicated, and the use is non-commercial. See: http://creativecommons.org/licenses/by-nc/4.0/.

\section{REFERENCES}

1 Stakenborg N, Gomez-Pinilla PJ, Boeckxstaens GE. Postoperative ileus: pathophysiology, current therapeutic approaches. Handb Exp Pharmacol 2017;239:39-57

2 Delaney CP. Clinical perspective on postoperative ileus and the effect of opiates. Neurogastroenterol Motil 2004;16(Suppl 2):61-6.

3 Traut U, Brügger L, Kunz R, et al. Systemic prokinetic pharmacologic treatment for postoperative adynamic ileus following abdominal surgery in adults. Cochrane Database Syst Rev 2008:CD004930.

4 Drake TM, Ward AE. Pharmacological management to prevent ileus in major abdominal surgery: a systematic review and meta-analysis. J Gastrointest Surg 2016:20:1253-64.

5 Kalff JC, Carlos TM, Schraut WH, et al. Surgically induced leukocytic infiltrates within the rat intestinal muscularis mediate postoperative ileus. Gastroenterology 1999;117:378-87.

6 Kalff JC, Schraut WH, Simmons RL, et al. Surgical manipulation of the gut elicits an intestinal muscularis inflammatory response resulting in postsurgical ileus. Ann Surg 1998;228:652-63. 
7 Kalff JC, Türler A, Schwarz NT, et al. Intra-abdominal activation of a local inflammatory response within the human muscularis externa during laparotomy. Ann Surg 2003;237:301-15.

8 Wehner S, Behrendt FF, Lyutenski BN, et al. Inhibition of macrophage function prevents intestinal inflammation and postoperative ileus in rodents. Gut 2007;56:176-85.

9 Pantelis D, Kabba MS, Kirfel J, et al. Transient perioperative pharmacologic inhibition of muscularis macrophages as a target for prophylaxis of postoperative ileus does not affect anastomotic healing in mice. Surgery 2010;148:59-70.

10 de Jonge WJ, van der Zanden EP, The FO, et al. Stimulation of the vagus nerve attenuates macrophage activation by activating the Jak2-STAT3 signaling pathway. Nat Immunol 2005;6:844-51.

11 Stakenborg N, Wolthuis AM, Gomez-Pinilla PJ, et al. Abdominal vagus nerve stimulation as a new therapeutic approach to prevent postoperative ileus. Neurogastroenterol Motil 2017;29:e13075.

12 Matteoli G, Gomez-Pinilla PJ, Nemethova A, et al. A distinct vagal anti-inflammatory pathway modulates intestinal muscularis resident macrophages independent of the spleen. Gut 2014;63:938-48.

13 Cailotto C, Costes LM, van der Vliet J, et al. Neuroanatomical evidence demonstrating the existence of the vagal anti-inflammatory reflex in the intestine. Neurogastroenterol Motil 2012;24:191-200. e93.

14 LePard KJ, Ren J, Galligan JJ. Presynaptic modulation of cholinergic and noncholinergic fast synaptic transmission in the myenteric plexus of guinea pig ileum. Neurogastroenterol Motil 2004;16:355-64.

15 De Maeyer JH, Lefebvre RA, Schuurkes JA. 5-HT4 receptor agonists: similar but not the same. Neurogastroenterol Motil 2008;20:99-112.

16 Grundmann D, Klotz M, Rabe $H$, et al. Isolation of high-purity myenteric plexus from adult human and mouse gastrointestinal tract. Sci Rep 2015:5:9226.

17 van Bree SH, Gomez-Pinilla PJ, van de Bovenkamp FS, et al. Inhibition of spleen tyrosine kinase as treatment of postoperative ileus. Gut 2013:62:1581-90.

18 van Bree SH, Nemethova A, van Bovenkamp FS, et al. Novel method for studying postoperative ileus in mice. Int J Physiol Pathophysiol Pharmacol 2012;4:219.

19 Wolthuis AM, Stakenborg N, D'Hoore A, et al. The pig as preclinical model for laparoscopic vagus nerve stimulation. Int J Colorectal Dis 2016;31:211-5.

20 Topal B, Fieuws S, Aerts R, et al. Pancreaticojejunostomy versus pancreaticogastrostomy reconstruction after pancreaticoduodenectomy for pancreatic or periampullary tumours: a multicentre randomised trial. Lancet Oncol 2013;14:655-62.

21 Adams DJ, Berecki G. Mechanisms of conotoxin inhibition of N-type (Ca(v)2.2) calcium channels. Biochim Biophys Acta 2013;1828:1619-28.

22 Briejer MR, Bosmans JP, Van Daele P, et al. The in vitro pharmacological profile of prucalopride, a novel enterokinetic compound. Eur J Pharmacol 2001;423:71-83.

23 The FO, Boeckxstaens GE, Snoek SA, et al. Activation of the cholinergic antiinflammatory pathway ameliorates postoperative ileus in mice. Gastroenterology 2007;133:1219-28.

24 Gabanyi I, Muller PA, Feighery L, et al. Neuro-immune interactions drive tissue programming in intestinal macrophages. Cell 2016;164:378-91.

25 Drisdel RC, Green WN. Neuronal alpha-bungarotoxin receptors are alpha7 subunit homomers. J Neurosci 2000;20:133-9.
26 Moretti M, Zoli M, George AA, et al. The novel $\alpha 7 \beta 2$-nicotinic acetylcholine receptor subtype is expressed in mouse and human basal forebrain: biochemical and pharmacological characterization. Mol Pharmacol 2014;86:306-17.

27 Qu H, Sun GR, Zhou SQ, et al. Clinical risk factors of delayed gastric emptying in patients after pancreaticoduodenectomy: a systematic review and meta-analysis. Eur $J$ Surg Oncol 2013;39:213-23.

28 Qu Y, Ren S, Li C, et al. Management of postoperative complications following splenectomy. Int Surg 2013;98:55-60.

29 Cailotto C, Gomez-Pinilla PJ, Costes LM, et al. Neuro-anatomical evidence indicating indirect modulation of macrophages by vagal efferents in the intestine but not in the spleen. PLoS One 2014;9:e87785.

30 Myrtek D, Müller T, Geyer V, et al. Activation of human alveolar macrophages via P2 receptors: coupling to intracellular $\mathrm{Ca} 2+$ increases and cytokine secretion. J Immunol 2008;181:2181-8.

31 Berthoud HR, Carlson NR, Powley TL. Topography of efferent vagal innervation of the rat gastrointestinal tract. Am J Physiol 1991;260:R200-7.

32 Priem E, Van Colen I, De Maeyer JH, et al. The facilitating effect of prucalopride on cholinergic neurotransmission in pig gastric circular muscle is regulated by phosphodiesterase 4. Neuropharmacology 2012;62:2126-35.

33 Tsuchida Y, Hatao F, Fujisawa M, et al. Neuronal stimulation with 5-hydroxytryptamine 4 receptor induces anti-inflammatory actions via $\alpha 7 \mathrm{nACh}$ receptors on muscularis macrophages associated with postoperative ileus. Gut 2011;60:638-47.

34 Narita K, Tsunoda A, Takenaka K, et al. Effect of mosapride on recovery of intestinal motility after hand-assisted laparoscopic colectomy for carcinoma. Dis Colon Rectum 2008;51:1692-5.

35 Toyomasu Y, Mochiki E, Morita H, et al. Mosapride citrate improves postoperative ileus of patients with colectomy. J Gastrointest Surg 2011;15:1361-7.

36 Galandiuk S, Beyens G, Ausma J, et al. 934 Evaluation of the efficacy, safety and tolerability of prucalopride (Resolor $®$ ) given subcutaneously in patients undergoing elective partial colectomies. Gastroenterology 2008;134:A-138.

37 Gong J, Xie Z, Zhang T, et al. Randomised clinical trial: prucalopride, a colonic pro-motility agent, reduces the duration of post-operative ileus after elective gastrointestinal surgery. Aliment Pharmacol Ther 2016;43:778-89.

38 Liu MT, Kuan YH, Wang J, et al. 5-HT4 receptor-mediated neuroprotection and neurogenesis in the enteric nervous system of adult mice. J Neurosci 2009;29:9683-99.

39 Kawahara I, Kuniyasu $\mathrm{H}$, Matsuyoshi $\mathrm{H}$, et al. Comparison of effects of a selective 5-HT reuptake inhibitor versus a 5-HT4 receptor agonist on in vivo neurogenesis at the rectal anastomosis in rats. Am J Physiol Gastrointest Liver Physiol 2012:302:G588-97.

40 Takaki M, Goto K, Kawahara I. The 5-hydroxytryptamine 4 receptor agonistinduced actions and enteric neurogenesis in the gut. J Neurogastroenterol Motil 2014;20:17-30.

41 Matsuyoshi H, Kuniyasu H, Okumura M, et al. A 5-HT(4)-receptor activation-induced neural plasticity enhances in vivo reconstructs of enteric nerve circuit insult. Neurogastroenterol Motil 2010;22:806-13. e226. 